\title{
Aprendizaje organizacional: una variable de madurez de la gestión de la seguridad y salud en el trabajo ${ }^{1}$
}

\author{
Yuber Liliana Rodríguez-Rojas ${ }^{2}$
}

\begin{abstract}
Resumen
El proyecto de investigación busca identificar si el aprendizaje organizacional es una variable que contribuye en la medición de la madurez de la gestión de la seguridad y salud en el trabajo en universidades con acreditación de alta calidad multicampus. Se efectuó una revisión de 37 artículos, recuperados de 4 bases de datos, para la conceptualización de las variables. Además, se realizó un estudio cuantitativo transversal con 1040 sujetos. La información fue procesada con spss usando el índice de correlación Rho de Spearman. Los resultados muestran que el aprendizaje organizacional tiene una correlación entre moderada y fuerte con las otras variables del contexto interno, como el análisis estratégico, la planeación estratégica, la planeación de la capacidad, la evaluación de la GSST y la participación y comunicación. Asimismo, mostró correlación entre fuerte y perfecta con la integración de la GSST en la organización. Se concluye que la variable aprendizaje organizacional sí aporta en la determinación del nivel de madurez de la GSST en la organización estudiada.
\end{abstract}

Palabras clave: aprendizaje organizacional, seguridad y salud en el trabajo, evaluación de la madurez.

Este texto presenta los resultados parciales del proyecto de investigación titulado Evaluación de la madurez de la gestión de la seguridad y salud en el trabajo en universidades privadas con acreditación de alta calidad multicampus de Colombia.

Líder de la línea de investigación en gestión en salud y docente investigadora de la Maestría en Calidad y Gestión Integral, convenio Universidad Santo Tomás-Icontec. Correo electrónico: yuberliliana@gmail.com, yuberrodriguez@usantotomas.edu.co 


\section{Introducción}

Colombia asumió el reto de pasar del paradigma de la salud ocupacional a la seguridad y salud en el trabajo. El cambio se basó en la actualización de la legislación en riesgos laborales efectuada en el 2012. Esta reforma exige la implementación de un sistema de gestión de la seguridad y salud en el trabajo (sGSST) en todas las organizaciones, independientemente de su tamaño y actividad económica, lo cual requiere del desarrollo de nuevas metodologías que permitan la implementación eficaz de este sistema de gestión en las organizaciones.

Adicionalmente, a partir de la Ley 1562 de 2012, los estudiantes que desarrollen prácticas académicas serán afiliados al Sistema General de Riesgos Laborales colombiano (SGRL), lo que requiere un mayor compromiso de las instituciones de educación superior con la GSST, dado que, si bien el afiliar a los practicantes al SGRL no constituye un vínculo contractual, sí se requiere incorporarlos a todas las acciones de seguridad y salud en el trabajo adelantadas, así como la medición del desempeño del SG-SST que evidencie el impacto de este sistema en la protección de la salud y el fomento de la seguridad, tanto de estudiantes como de docentes, personal administrativo, contratistas, proveedores, visitantes, entre otros.

En este sentido, es imperativa la necesidad de desarrollar métodos complementarios para la evaluación de la GSST en las instituciones de educación superior. Particularmente, las instituciones con acreditación de alta calidad multicampus requieren ser pioneras en la evaluación de la madurez de la GSST por considerarse las organizaciones con niveles deseables de calidad que responden a su naturaleza y carácter. Para las organizaciones, contar con información válida y confiable en seguridad y salud en el trabajo contribuirá al diagnóstico y a la toma de decisiones en materia de su gestión.

Si bien existen investigaciones que describen métodos de evaluación de la gestión de la seguridad y salud en el trabajo (GSST) (Fernández, Montes y Vásquez, 2006; Favaro y Drais, 2007; Saracino et ál, 2012; Robson, Macdonald, Gray, Van Eerd y Bigelow, 2012; Rodríguez y Molano, 2012; Saracino et ál., 2015; Arntz-Gray, 2016; Kim, Park y Park, 2016), así como indicadores que miden el desempeño de los SG-ssT (Mohammadfam, Kamalinia, Momeni, Golmohammadi, Hamidi y Soltanian, 2017) y para Colombia los estándares mínimos de GSST establecidos en la Resolución 1111 de 2017, son pocos los estudios en los que se habla de madurez de la GSST. 
En este sentido, se requiere de la identificación de variables que permitan medir el desempeño de los SG-SST y su madurez de forma objetiva. De acuerdo con lo descrito por Rodríguez-Rojas, Pedraza-Nájar y Martínez (2017), para determinar la madurez se han creado distintos modelos:

[...] los cuales indican las capacidades [competencia específica que debe existir en una organización con el fin de que se ejecuten procesos de gestión de proyectos y entregar servicios de gestión de proyectos y productos] de una organización. Estas capacidades se presentan de forma agrupada y describen generalmente el grado de coherencia, la visibilidad y el control dentro de la organización. (p. 4)

A partir de la revisión de la literatura se encontró que una de las variables que permiten medir la madurez de la GSST es el aprendizaje y el desarrollo de la cultura de los individuos. Esto atiende a que las organizaciones se enfrentan a nuevos retos en su gestión para poder obtener los resultados deseados.

De acuerdo con Weick y Sutcliffe (2015), los tomadores de decisiones y las organizaciones necesitan desarrollar capacidades para una mayor atención en el conocimiento de detalles que provean información relevante para la toma de decisiones y, particularmente, aquella información que provea elementos para crear orden y fomentar la previsibilidad dentro de la organización. Los autores plantearon cinco aspectos esenciales: 1) la preocupación por el fracaso, 2) la reticencia para simplificar, 3) la sensibilidad frente a las operaciones, 4) el compromiso con la capacidad de recuperación, y 5) la deferencia a la experiencia.

Se ha demostrado que uno de los aspectos que mejora el rendimiento de las organizaciones es la satisfacción de sus trabajadores. Por consiguiente, otro de los retos para las organizaciones es aprender a utilizar la experiencia del pasado para mejorar la salud y la seguridad de sus trabajadores, por ejemplo, a través del aprendizaje de los incidentes y accidentes.

En el estudio Drupsteen y Wybo (2015), su objetivo fue desarrollar un conjunto de indicadores para la propensión a aprender, como parte de los principales indicadores de seguridad. Para ello, se midió la percepción individual de aprendizaje de la experiencia a través de un conjunto de indicadores. Los indicadores fueron validados a través de entrevistas en un centro de producción francés.

En esta investigación se plantearon dos series de indicadores. La primera serie de indicadores es organizacional: están orientados a la identificación de las fortalezas, debilidades y formas de mejora como parte de los principales indicadores de seguridad. La segunda serie de indicadores son del nivel individual: 
estos propenden por la identificación de las personas que puedan desempeñar el papel de "agentes de aprendizaje", promoviendo un proceso en su área para identificar grupos de personas que necesitan una formación específica para mejorar su voluntad, actitud o las habilidades para lograr las diferentes tareas del proceso de aprendizaje.

Los indicadores organizacionales propuestos por Drupsteen y Wybo (2015) son seis: dos indicadores destinados a medir la actitud hacia el aprendizaje, dos indicadores dirigidos a medir las condiciones de organización para el aprendizaje y dos indicadores destinados a determinar la disponibilidad de sistemas que facilitan el aprendizaje. Por otra parte, los autores utilizaron cinco indicadores para determinar la propensión individual del aprendizaje: actitud para detectar, actitud para analizar, actitud para el seguimiento, la actitud de la evaluación, y la actitud de compartir información (Figura 1).

Los indicadores propuestos permitieron apoyar la identificación de debilidades con respecto al aprendizaje a nivel organizativo y facilitaron la identificación de las necesidades de formación de los trabajadores.

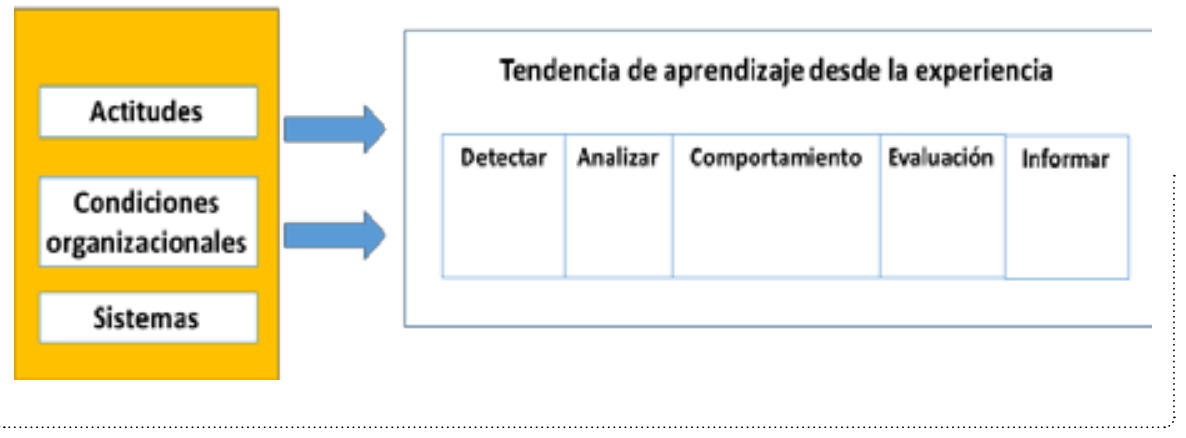

Figura 1. Modelo para estudiar las tendencias de aprendizaje desde la experiencia.

(Fuente: Drupsteen y Wybo, 2015. Traducción propia)

Otro de los modelos propuestos para explicar la propensión del esfuerzo es el desarrollado por Schnake (2007), quien identificó siete efectos positivos directos: satisfacción en el trabajo, capacidad de trabajo, compromiso con la organización, rasgos de personalidad, capacidad para realizar tareas relacionadas con el trabajo, normas de comportamiento y tamaño del grupo, y la moderación por el temor a la evaluación.

El fin del aprendizaje en la organización es la creación de una cultura, en este caso de una cultura de seguridad, la cual es concebida, de acuerdo con la International Atomic Energy Agency, como el conjunto de características y acti- 


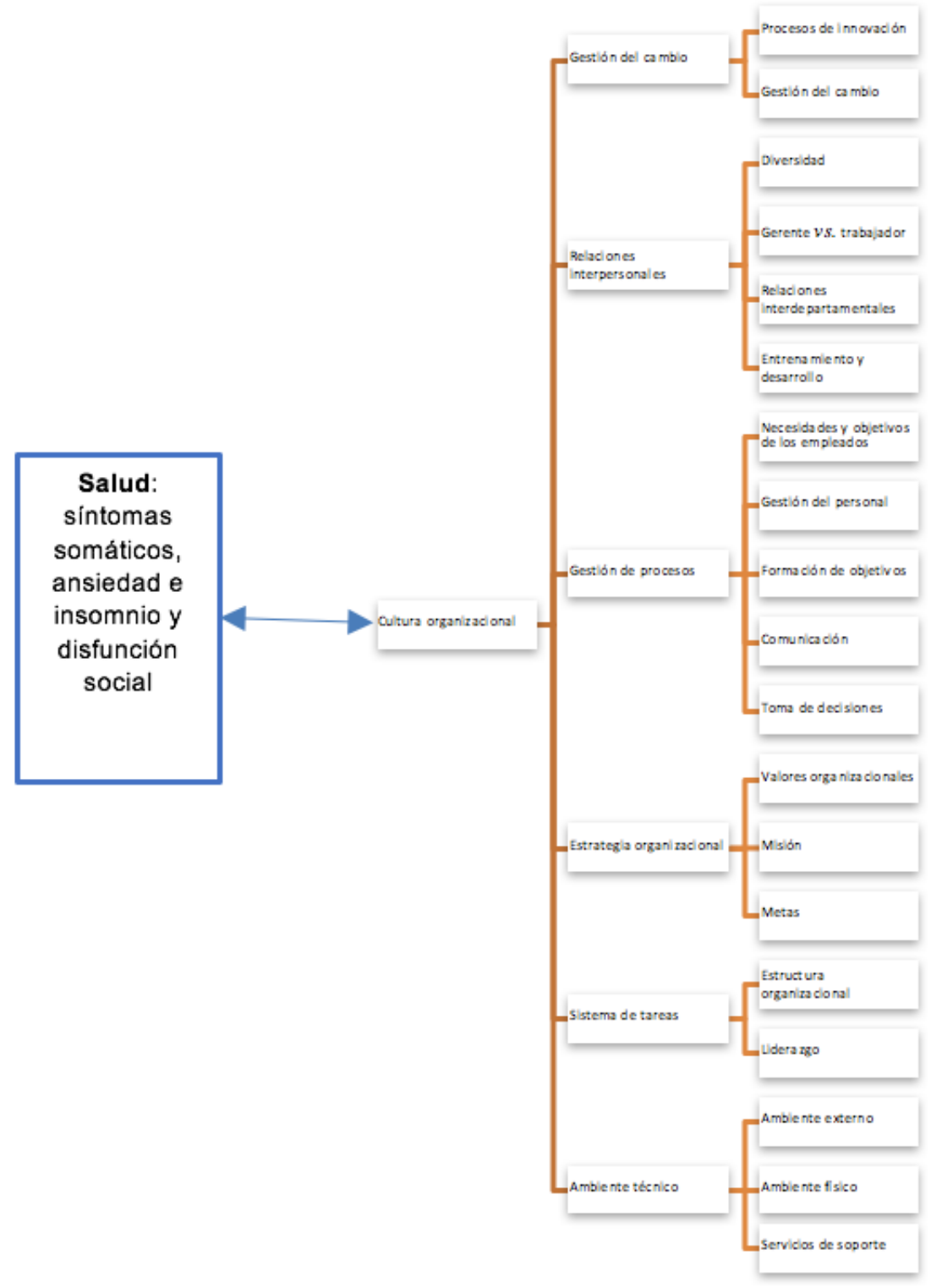

Figura 2. Modelo teórico de cultura organizacional y salud ocupacional.

[Fuente: Martins y Nel, 2014. Traducción propia) 
tudes en las organizaciones e individuos, que, a su vez, son establecidos como prioridad esencial. La cultura de seguridad débil ha sido una de las causas de sucesos como Bhopal, por cuanto el nivel de cultura de seguridad tiene que ser evaluado y mejorado para evitar la ocurrencia de este tipo de situaciones en el futuro de forma continua (Warszawska y Kraslawski, 2015).

Otra definición de cultura organizacional es presentada por Martins y Coetzee (2007). Es un patrón integrado de comportamiento, que es único para una organización particular y que se originó como resultado del proceso de la supervivencia y la interacción con su entorno. Además, la cultura dirige la organización para el logro de metas.

Esta conceptualización dio origen a un modelo teórico de cultura organizacional y salud ocupacional (Figura 2), validado a través del modelo de ecuaciones estructurales. El modelo explica que la salud puede tener un impacto en la cultura organizacional y viceversa (Martins y Nel, 2014). En este sentido, la seguridad y salud en el trabajo cobra importancia en la cultura organizacional y en el logro de las metas de la organización.

Atendiendo a la necesidad de identificar las variables que permiten medir la madurez de la GSST en las organizaciones, en esta investigación se buscó desarrollar teóricamente y verificar empíricamente si el aprendizaje organizacional se constituye como una variable latente en la dimensión de contexto interno al momento de medir la madurez de esta gestión en universidades privadas colombianas con acreditación de alta calidad multicampus.

\section{Metodología}

Se realizó una investigación de tipo cuantitativo, puesto que se buscó confirmar si el aprendizaje organizacional es una de las variables latentes que se debe incluir en la dimensión de contexto interno de un instrumento para medir la madurez de la GSST en universidades privadas colombianas con acreditación de alta calidad multicampus.

La investigación tuvo un alcance explicativo porque parte de comprender las teorías existentes relacionadas con la GSST y su madurez. A partir de estas se define si el aprendizaje organizacional puede ser considerado como una variable del contexto interno, el cual hace parte de las variables latentes de un instrumento para medir la madurez de la GSST, dirigido a universidades privadas con acreditación de alta calidad.

En relación con los diseños de investigación cuantitativos, corresponde a un estudio no experimental de corte transversal puesto que se partió de una revi- 
sión sistemática de la literatura científica y, posteriormente, se recopiló una serie de datos en el escenario de estudio, en un momento determinado en el tiempo.

Como unidad de análisis se tomaron las instituciones con sede principal en la ciudad de Bogotá, Colombia, que cuentan con acreditación multicampus de alta calidad otorgada por el Consejo Nacional de Acreditación (CNA), debido a la madurez administrativa de las instituciones de la capital que cuentan con este reconocimiento, el cual es sinónimo de calidad y prestigio.

Cabe aclarar que una institución de educación superior, para contar con acreditación multicampus bajo los nuevos lineamientos - establecidos para Colombia en el 2015-, deberá acreditar el $100 \%$ de las sedes y seccionales. Además, debe tener programas acreditados en al menos el $75 \%$ de sus sedes o seccionales, donde deberá contar con al menos el $10 \%$ de su oferta académica acreditada (CNA, 2015). Este reconocimiento también indica un reflejo de unidad para aquellas universidades que cuentan con gran cantidad de sedes a lo largo del territorio nacional, sin desconocer las necesidades específicas de cada una según el lugar donde se encuentren.

De acuerdo con datos del sniEs, son 5 universidades las que cuentan con este reconocimiento. Por otra parte, debido a las diferencias en la gestión que tienen las universidades públicas y privadas, se tomó como unidad de muestreo su carácter jurídico. En este sentido, son 4 las instituciones de educación superior privadas con sede en Bogotá que cuentan con acreditación de alta calidad multicampus, de acuerdo con el SNIEs.

El escenario de estudio fue seleccionado por conveniencia debido a las restricciones que se tienen para acceder a información de SST en las organizaciones, de modo que se tomó la primera institución de educación superior de carácter privado acreditada institucionalmente en la modalidad multicampus.

La institución de educación superior analizada cuenta con 2661 docentes, 1489 administrativos, 32535 estudiantes y 143505 egresados, para un total de 36684 personas (dato recopilado el 23 de noviembre de 2016). Para seleccionar los sujetos de investigación, se efectuó un muestreo aleatorio de los grupos de interés. Se calculó una muestra de 1037 sujetos de investigación.

En relación con las técnicas de investigación empleadas, se realizó una revisión sistemática de artículos publicados entre 2011 y febrero de 2017 en Science Direct, Ebsco, Eric y Redalyc, con los términos clave "maturity", "occupational health and safety management", "occupational health and safety management systems", "safety and health at work". Esta revisión permitió detectar 160 artículos, de los cuales 37 aportaron a la construcción teórica de las variables de contexto interno para la medición de la Gsst (Rodríguez-Rojas, Pedraza-Nájar y Martínez, 
2017). La revisión teórica efectuada permitió establecer las variables del contexto interno organizacional que influyen en la madurez de la Gsst (Tabla 1).

\section{Tabla 1. Variables teóricas del contexto interno organizacional que influyen en la madurez de la GSST}

\begin{tabular}{|c|c|}
\hline $\begin{array}{l}\text { Variable teórica para la } \\
\text { medición de la madurez de la } \\
\text { GSST o variable latente }\end{array}$ & Variables \\
\hline \multirow{6}{*}{ Contexto interno de la organización } & Aprendizaje organizacional en GSST \\
\hline & Análisis estratégico \\
\hline & Participación y comunicación \\
\hline & Evaluación de la GSST \\
\hline & Planeación estratégica \\
\hline & Planeación de la capacidad \\
\hline
\end{tabular}

Se planteó la siguiente hipótesis: el aprendizaje organizacional tiene una correlación moderada con las siguientes variables: análisis estratégico, planeación estratégica, planeación de la capacidad, integración de la GSST en la organización, evaluación de la GSST, participación y comunicación.

Las variables enunciadas hacen parte de la dimensión de contexto interno al momento de medir la madurez de la GSST en universidades privadas colombianas con acreditación de alta calidad multicampus.

Considerando la propuesta teórica de variables, se propuso un instrumento al cual se le realizó una validación de contenido a partir de la consulta a expertos, y validación de constructo a través de análisis de componentes principales y de la rotación de los datos, utilizando el método de Normalización Varimax con Kaiser. Se utilizó el índice de correlación Rho de Spearman, calculado con SPSS, para determinar si la variable denominada aprendizaje organizacional aporta significativamente en la dimensión de contexto interno al momento de medir la madurez de la GSST en universidades privadas colombianas con acreditación de alta calidad multicampus.

Las variables relacionadas en la Tabla 1 fueron incorporadas en un instrumento que consideró tres variables latentes: contexto interno, contexto externo y enfoque de la seguridad y salud en el trabajo. El instrumento diseñado obtuvo un Alfa de Cronbach de 0,967, de lo cual se puede deducir que es un test excelente y que mide el constructo propuesto. A su vez se aplicó la curva de Cronbach Mesbah, en la cual se calcula el coeficiente removiendo ítem por ítem. Se puede a observar que la fiabilidad no aumenta significativamente si se quitan ítems, lo que significa que el test está bien construido. 
Además, el instrumento fue sometido a pruebas más rigurosas, como el test de esfericidad de Bartlett y el кмо. Se obtuvo un кмо de 0,969, el cual es cercano a 1, y la prueba de esfericidad de Bartlett tiene significancia que tiende a 0. Lo anterior indica que las matrices de correlación fueron adecuadas para el análisis factorial.

\section{Resultados}

El instrumento diseñado para medir la madurez de la GSST fue aplicado a 1040 sujetos del escenario de estudio, aspecto que indica que se alcanzó la muestra esperada (Tabla 2).

Tabla 2. Distribución de la población analizada

\begin{tabular}{|l|c|c|}
\hline \multicolumn{1}{|c|}{ Población } & $\begin{array}{c}\text { Número de } \\
\text { personas }\end{array}$ & $\%$ \\
\hline Actor & & \\
\hline Administrativo & 326 & $31,3 \%$ \\
\hline Docente & 272 & $26,4 \%$ \\
\hline Estudiante de posgrado & 44 & $4,2 \%$ \\
\hline Estudiante de pregrado & 398 & $38,3 \%$ \\
\hline Ciudad/modalidad & & \\
\hline Tunja - modalidad presencial & 45 & $4,3 \%$ \\
\hline Bogotá - modalidad presencial & 456 & $43,8 \%$ \\
\hline Bucaramanga - modalidad presencial & 206 & $19,8 \%$ \\
\hline Medellín - modalidad presencial & 2 & $0,2 \%$ \\
\hline Villavicencio - modalidad presencial & 246 & $23,7 \%$ \\
\hline Educación virtual & 85 & $8,2 \%$ \\
\hline
\end{tabular}

\section{Aprendizaje organizacional en GSST}

A partir de la aplicación del instrumento a los 1040 sujetos de investigación, se analizó la variable de aprendizaje organizacional. La escala de evaluación fue de 1 a 5 y, considerando las respuestas, se hablará de Top two boxes (ттв): suma de porcentajes que calificaron 4 y 5 . De Bottom two boxess (втв) es suma de los porcentajes de los dos valores menores de la escala 1 y 2 . 
El aprendizaje organizacional tiene un alto ттв, dado que el 57,4 \% de la población encuestada manifiesta que la institución de educación superior cuenta con estrategias para garantizar el aprendizaje organizacional en Ssт. Se observó una baja proporción de duda (Figura 3).

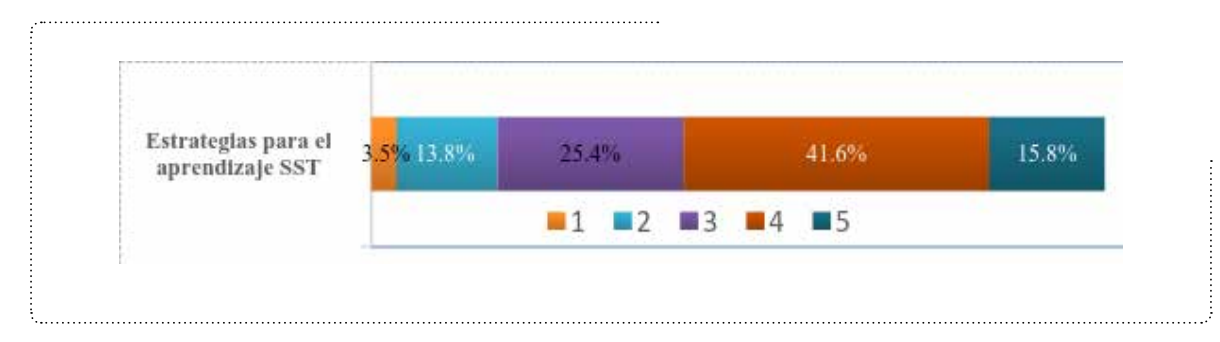

Figura 3. Aprendizaje organizacional en GSST.

*1) Está totalmente en desacuerdo, 2) Está en desacuerdo, 3) Ni de acuerdo, ni en desacuerdo, 4) Está de acuerdo, 5) Está totalmente de acuerdo.

Además, se encontraron correlaciones estadísticamente significativas. Estas correlaciones fueron calculadas a través de coeficiente Rho de Spearman y, para su interpretación, se usó la escala de rango de relación (Martínez, Tuya, Martínez, Pérez y Cánovas, 2009):

0 - 0,25: Escasa o nula

0,26 - 0,50: Débil

0,51 - 0,75: Entre moderada y fuerte

0,76 - 1,00: Entre fuerte y perfecta

La variable aprendizaje organizacional mostró una correlación entre moderada y fuerte con las otras variables del contexto interno, como el análisis estratégico, planeación estratégica y planeación de la capacidad (Tabla 3), lo que evidencia la importancia de la sistematización y evaluación del aprendizaje en seguridad y salud en el trabajo alcanzado por los actores (también conocidos como partes interesadas) de la institución de educación superior estudiada, en la definición de sus estrategias organizacionales. 
Tabla 3. Correlaciones Rho Spearman. Variables latentes análisis estratégico, planeación estratégica, planeación de la capacidad

y aprendizaje organizacional en sst

\begin{tabular}{|c|c|c|c|c|c|c|}
\hline & $\begin{array}{c}\text { Aprendizaje } \\
\text { organizacional }\end{array}$ & $\begin{array}{c}\text { Análisis } \\
\text { estratégico }\end{array}$ & $\begin{array}{l}\text { Planeación } \\
\text { estratégica }\end{array}$ & $\begin{array}{l}\text { Planeación de } \\
\text { la capacidad }\end{array}$ \\
\hline \multirow{12}{*}{ 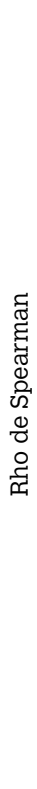 } & \multirow[t]{2}{*}{ 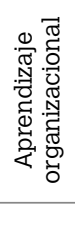 } & $\begin{array}{l}\text { Coeficiente de } \\
\text { correlación }\end{array}$ & 1,000 &, $683^{* *}$ &, $661^{* *}$ &, $600^{* *}$ \\
\hline & & Sig. (bilateral) & . & ,000 & ,000 & ,000 \\
\hline & \multirow{3}{*}{ 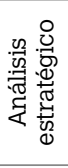 } & $\mathrm{N}$ & 1040 & 1040 & 1040 & 1040 \\
\hline & & $\begin{array}{l}\text { Coeficiente de } \\
\text { correlación }\end{array}$ &, $683^{* *}$ & 1,000 & $669^{* *}$ &, $607^{* *}$ \\
\hline & & Sig. (bilateral) & ,000 & . &, 000 &, 000 \\
\hline & \multirow{3}{*}{ 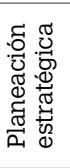 } & $\mathrm{N}$ & 1040 & 1040 & 1040 & 1040 \\
\hline & & $\begin{array}{l}\text { Coeficiente de } \\
\text { correlación }\end{array}$ & $661^{* *}$ & $669 * *$ & 1,000 & $667^{* *}$ \\
\hline & & Sig. (bilateral) & ,000 &, 000 & & ,000 \\
\hline & \multirow{4}{*}{ 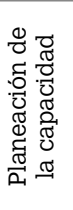 } & $\mathrm{N}$ & 1040 & 1040 & 1040 & 1040 \\
\hline & & $\begin{array}{l}\text { Coeficiente de } \\
\text { correlación }\end{array}$ &, $600^{* *}$ &, $607^{* *}$ & $667^{* *}$ & 1,000 \\
\hline & & Sig. (bilateral) &, 000 &, 000 &, 000 & . \\
\hline & & $\mathrm{N}$ & 1040 & 1040 & 1040 & 1040 \\
\hline
\end{tabular}

** La correlación es significativa al nivel 0,01 (bilateral).

Los resultados también mostraron que la variable "aprendizaje organizacional" tiene una correlación entre fuerte y perfecta con la variable "integración de la GSsT en la organización” (Tabla 4), la cual está compuesta por dos indicadores: 1) la organización tiene estrategias definidas para la mejora continua de la GSST y 2) la GSST está integrada en la gestión global de la organización, en la gestión estratégica y en el sistema de gobierno de la organización.

Además, mostró una correlación moderada y fuerte con las variables "participación y comunicación” y "evaluación de la GSST" (Tabla 4). 
Tabla 4. Correlaciones Rho Spearman.

Otras variables con el aprendizaje organizacional en SST

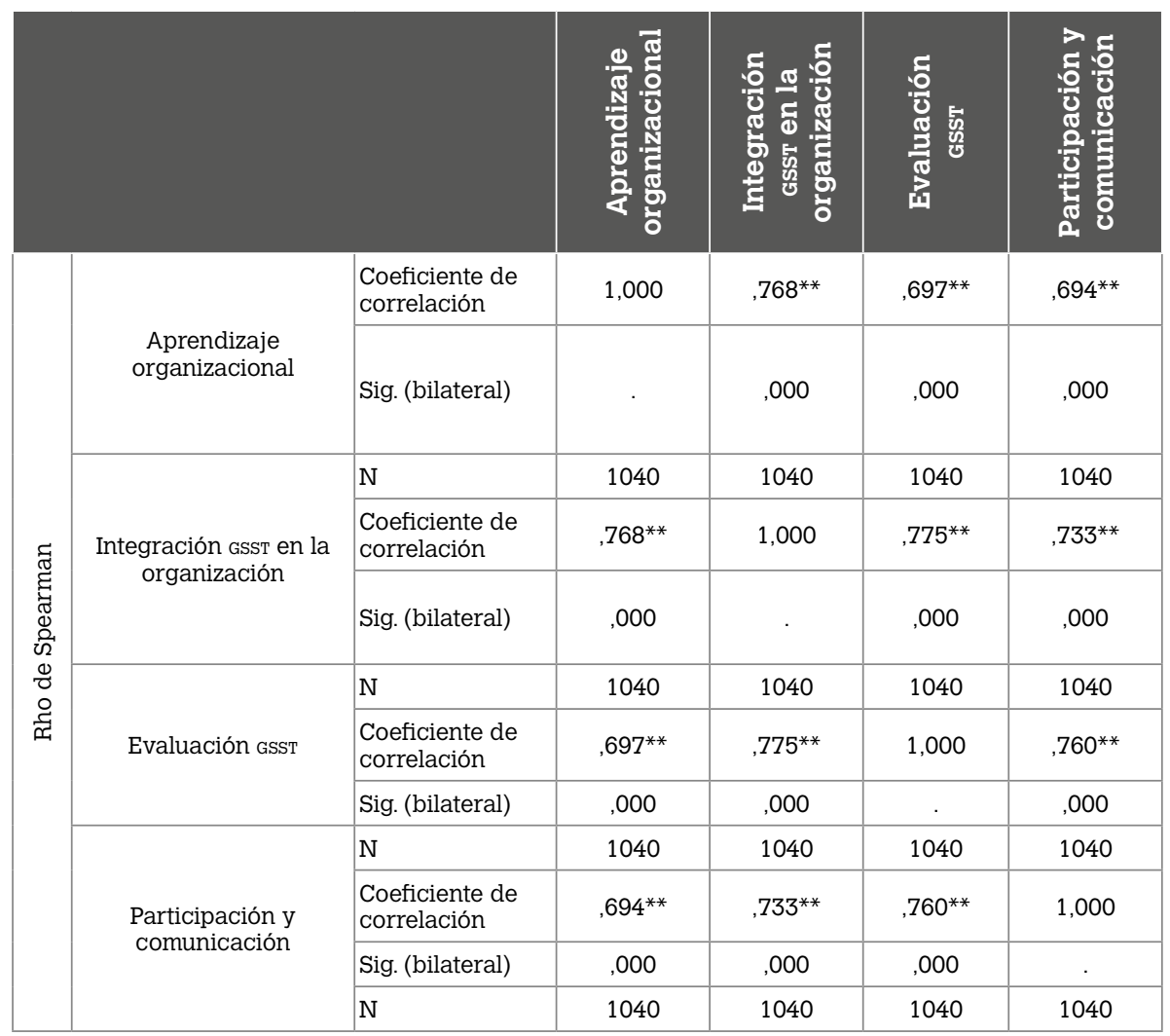

** La correlación es significativa al nivel 0,01 (bilateral).

Lo anterior permitió verificar la hipótesis planteada y se evidenció que el aprendizaje organizacional tiene una correlación entre moderada y fuerte con las variables "análisis estratégico", "planeación estratégica”, "planeación de la capacidad”, "integración de la GSST en la organización”, "evaluación de la GSST" y “participación y comunicación”.

Con la investigación se logró determinar que el aprendizaje organizacional (aporta en un 4,6 \%) es una de las variables del contexto interno de la organización, el cual aporta un peso de 57,6 \% de la escala para determinar el grado de madurez de la GSST en universidades privadas colombianas con acreditación de alta calidad multicampus. 


\section{Conclusiones}

Se concluye que la variable "aprendizaje organizacional" sí aporta en la determinación del nivel de madurez de la GSST en la organización estudiada. En este sentido, las instituciones de educación superior requieren de la incorporación de estrategias para el fortalecimiento del aprendizaje organizacional en seguridad y salud en el trabajo. Como señalan Drupsteen y Wybo (2015), es necesario analizar a través de indicadores la propensión a aprender de los actores en SsT, logrando con ello identificar las actitudes de las personas, las condiciones organizacionales y los sistemas que favorecen la detección, el análisis, el comportamiento, la evaluación y la información de la tendencia de aprendizaje desde la experiencia.

En concreto, las empresas deben innovar en el diseño, implementación y mantenimiento de sus estructuras y sistemas de gestión del conocimiento para contribuir al logro de sus resultados (Nowacki y Bachnik, 2016). Esto requiere hacer uso del aprendizaje organizacional. Según Weick y Sutcliffe (2015), los tomadores de decisiones necesitan desarrollar capacidades que les permitan conocer detalles e información relevante para crear orden y fomentar la previsibilidad.

Es de precisar que el fin del "aprendizaje organizacional" es la creación de una cultura, puesto que una cultura de seguridad débil conlleva a accidentes graves (Warszawska y Kraslawski, 2015). De acuerdo con Martins y Coetzee (2007), la cultura organizacional es un patrón integrado de comportamiento, el cual dirige la organización al logro de metas. Según Martins y Nel (2014), la seguridad y salud en el trabajo puede tener un impacto en la cultura organizacional y viceversa.

El papel que desempeña el factor humano en la génesis de los accidentes e incidentes es indiscutible, sin embargo, no es el único factor. Por ende, el abordaje de la seguridad y salud en el trabajo debe darse de manera integral, considerando factores ambientales, condiciones de la operación, factores del personal, factores relacionados con la supervisión y factores de influencia organizacional, como los procesos, el clima organizacional, los recursos para la gestión (Ergai, Cohen, Sharp, Wiegmann, Wiegmann y Shappell, 2016) y la cultura de la organización.

Además, la evaluación de la cultura de seguridad de la organización puede ser una herramienta útil, dado que permite aprender sobre su cultura actual y comenzar a desarrollar enfoques de la SsT más proactivos (Haslam et ál., 2016).

De igual manera, se requiere estudiar otros aspectos como el liderazgo y la motivación, ya que el líder debe potenciar actitudes y crear una sensación de logro y de pertenencia a la organización, así como motivación, la cual es esencial 
para el cambio en la conducta de los trabajadores (Hedlund, Gummesson, Ryde$1 \mathrm{l}$ y Andersson, 2016). Estos son aspectos indispensables para la generación de cultura en seguridad y salud en el trabajo en las organizaciones.

Por otra parte, se requiere desarrollar métodos para la formulación, medición y evaluación de indicadores de GssT en las instituciones de educación superior, aspecto que ha sido poco estudiado y desarrollado desde la investigación organizacional. Esto se observó en la revisión de la literatura y en la medición de la GSST realizada en el escenario estudiado.

Además, se requiere el desarrollo de instrumentos complementarios que incluyan criterios de objetivación para evaluar las estrategias de aprendizaje organizacional en SST incorporados dentro de la GSST en las instituciones de educación superior. Este aspecto permitiría trascender los requisitos legales y los estándares mínimos establecidos en la normatividad colombiana y orientar la GSST hacia una cultura de prevención en ssT que aproveche la experiencia y el aprendizaje acumulados a partir de las prácticas de ssT.

\section{Referencias}

Arntz-Gray, J. (2016). Plan, Do, Check, Act: The need for independent audit of the internal responsibility system in occupational health and safety. Safety Science, $84,12-23$.

CNA (2015). Nuevos lineamientos de acreditación institucional. 2015. Bogotá, Colombia: Consejo Nacional de Acreditación. Recuperado de: http://www.cna.gov. co/1741/article-186359.html

Colombia, Congreso de la República. Ley 1562. Por la cual se modifica el Sistema de Riesgos Laborales y se dictan otras disposiciones en materia de Salud Ocupacional (11 julio 2012).

Colombia, Ministerio del Trabajo. (2017). Resolución 1111. Por la cual se definen los Estándares Mínimos del Sistema de Gestión de Seguridad y Salud en el Trabajo para emprendedores y contratantes (27 marzo 2017).

Colombia, Presidencia de la Drupsteen, L. y Wybo, J. (2015). Assessing propensity to learn from safety-related events. Safety Science, 71, 28-38. doi:10.1016/j. ssci.2014.02.024.

Ergai, E., Cohen, T., Sharp, J., Wiegmann, D., Wiegmann, A. y Shappell, S. (2016). Assessment of the Human Factors Analysis and Classification System (HFACS): Intra-rater and inter-rater reliability. Safety Science, 82, 393-398. doi: 10.1016/j. ssci.2015.09.028. 
Favaro, M. y Drais, E. (2007). Implementación de los sistemas de gestión de salud y seguridad en el trabajo. En Fifth International Conference on Occupational Risk Prevention (pp. 1-27). Santiago de Chile, Chile.

Fernández, B., Montes, J. y Vázquez, C. (2006). Desarrollo y validación de una escala de medición para el sistema de gestión de la seguridad laboral. Investigaciones Europeas de Dirección y Economía de la Empresa, 12(3), 77-93.

Haslam, C., O’Hara, J., Kazi, A., Twumasi, R. y Haslam, R. (2016). Proactive occupational safety and health management: Promoting good health and good business. Safety Science, 81, 99-108. doi: 10.1016/j.ssci.2015.06.010.

Hedlund, A., Gummesson, K., Rydell, A. y Andersson, M. (2016). Safety motivation at work: Evaluation of changes from six interventions. Safety Science, 82, 155163.

Kim, Y., Park, J. y Park, M. (2016). Creating a Culture of Prevention in Occupational Safety and Health Practice. Safety and Health at Work, 7(2), 89-96.

Martínez, R., Tuya, L., Martínez, M., Pérez, A. y Cánovas, A. (2009). El coeficiente de correlación de los rangos de spearman caracterización. Revista Habanera de Ciencias Médicas, 8(2), 1-19.

Martins, N. y Nel, M. (2014). Validating a Theoretical Model of Organisational Culture and Occupational Health by Means of Structural Equation Modelling. En Proceedings of the 13th European Conference on Research Methodology for Business and Management Studies (pp. 276). Londres, Inglaterra: Academic Conferences Limited.

Mohammadfam I, Kamalinia M, Momeni M, Golmohammadi R, Hamidi Y, Soltanian A. (2017). Evaluation of the quality of occupational health and safety management systems based on key performance indicators in Certified organizations. Safety and Health at Work, 8(2), 156-161. doi: 2048/10.1016/j. shaw.2016.09.001.

Nowacki, R. y Bachnik, K. (2016). Innovations within knowledge management. Journal of Business Research, 69(5), 1577-1581. doi.org/10.1016/j.jbusres.2015.10.020.

República. (2015). Decreto 1072. Por medio del cual se expide el Decreto Único Reglamentario del Sector Trabajo de 2015 (26 mayo 2015)

Robson, L., Macdonald, S., Gray, G., Van Eerd, D. y Bigelow, P. (2012). A descriptive study of the OHS management auditing methods used by public sector organizations conducting audits of workplaces: Implications for audit reliability and validity. Safety Science, 50(2), 181-189. doi:10.1016/j.ssci.2011.08.006. 
Rodríguez, Y. y Molano, J. (2012). Adaptación de una herramienta para la evaluación de la gestión de la salud y seguridad en el trabajo. El Hombre y la Máquina, 40, 7-21.

Rodríguez-Rojas, Y., Pedraza-Nájar, X., y Martínez, J. (2017). Evaluación de la madurez de la gestión de la seguridad y salud en el trabajo: Revisión de literatura. Revista Signos-Investigación en sistemas de gestión (en prensa).

Saracino, A., Antonioni, G., Spadoni, G., Guglielmi, D., Dottori, E., Flamigni, L., Malagoli, M. y Pacini, V. (2015). Quantitative assessment of occupational safety and health: Application of a general methodology to an italian multi-utility company. Safety Science, 72, 75-82. doi: 10.1016/j.ssci.2014.08.007.

Saracino, A., Spadoni, G., Curcuruto, M., Guglielmi, D., Bocci, V., Cimarelli, M., Dottori, E. y Violante, F. (2012). A New Model for Evaluating Occupational Health and Safety Management Systems (OHSMS). Chemical Engineering Transactions, 26, 519-524. doi: 10.3303/CET1226087.

Schnake, S. (2007). An integrative model of effort propensity. Human Resource Management Review, 17, 274-289. doi: 10.1016/j.hrmr.2007.07.003.

Warszawska, K. y Kraslawski, A. (2015). Method for quantitative assessment of safety culture. Journal of Loss Prevention in the Process Industries, 42, 27-34. doi. org/10.1016/j.jlp.2015.09.005.

Weick, K. y Sutcliffe, K. (2015). Managing the Unexpected: Sustained Performance in a Complex World. Nueva Jersey, Estados Unidos: John Wiley-Sons, Inc. 